Acta Crystallographica Section D

Biological

Crystallography

ISSN 0907-4449

Editors: E. N. Baker and Z. Dauter

\title{
Structure determination from a single high-pressure-frozen virus crystal
}

\author{
Anja Burkhardt, Armin Wagner, Martin Warmer, Rudolph Reimer, \\ Heinrich Hohenberg, Jingshan Ren, Elizabeth E. Fry, David I. Stuart and \\ Alke Meents
}

Acta Cryst. (2013). D69, 308-312

Copyright (C) International Union of Crystallography

Author(s) of this paper may load this reprint on their own web site or institutional repository provided that this cover page is retained. Republication of this article or its storage in electronic databases other than as specified above is not permitted without prior permission in writing from the IUCr.

For further information see http://journals.iucr.org/services/authorrights.html

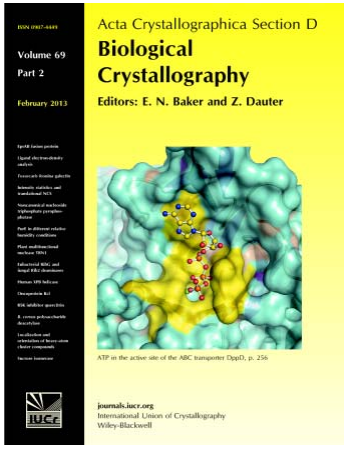

\begin{abstract}
Acta Crystallographica Section D: Biological Crystallography welcomes the submission of papers covering any aspect of structural biology, with a particular emphasis on the structures of biological macromolecules and the methods used to determine them. Reports on new protein structures are particularly encouraged, as are structure-function papers that could include crystallographic binding studies, or structural analysis of mutants or other modified forms of a known protein structure. The key criterion is that such papers should present new insights into biology, chemistry or structure. Papers on crystallographic methods should be oriented towards biological crystallography, and may include new approaches to any aspect of structure determination or analysis. Papers on the crystallization of biological molecules will be accepted providing that these focus on new methods or other features that are of general importance or applicability.
\end{abstract}

Crystallography Journals Online is available from journals.iucr.org 
Acta Crystallographica Section D

Biological

Crystallography

ISSN 0907-4449
Anja Burkhardt, ${ }^{a}$ Armin Wagner, ${ }^{\text {b }}$ Martin Warmer, ${ }^{c}$ Rudolph Reimer, ${ }^{\mathrm{c}}$ Heinrich Hohenberg, Jingshan Ren, Elizabeth E. Fry, ${ }^{d}$ David I. Stuart $^{\mathrm{b}, \mathrm{d}}$ and Alke Meents ${ }^{\mathrm{a} *}$

a Deutsches Elektronen-Synchrotron (DESY), Notkestrasse 85, 22607 Hamburg, Germany, ${ }^{\mathbf{b}}$ Diamond Light Source, Chilton, Didcot, Oxfordshire OX11 ODE, England, ${ }^{\mathbf{c}}$ Heinrich Pette Institute, Leibniz Institute for Experimental Virology, Martinistrasse 52, 20251 Hamburg, Germany, and Division of Structural Biology, University of Oxford, The Henry Wellcome Building for Genomic Medicine, Headington, Oxford OX3 7BN, England

Correspondence e-mail: alke.meents@desy.de

Received 22 August 2012

Accepted 2 November 2012
(C) 2013 International Union of Crystallography Printed in Singapore - all rights reserved

\section{Structure determination from a single high-pressure-frozen virus crystal}

Successful cryogenic X-ray structure determination from a single high-pressurefrozen bovine enterovirus 2 crystal is reported. The presented high-pressurefreezing procedure is based on a commercially available device and allows the cryocooling of macromolecular crystals directly in their mother liquor without the time- and crystal-consuming search for optimal cryoconditions. The method is generally applicable and will allow cryogenic data collection from all types of macromolecular crystals.

\section{Introduction}

A major limitation to successful crystal structure determination in macromolecular crystallography (MX), especially for large molecular complexes, is X-ray-induced radiation damage (Blake \& Phillips, 1962). Cryocooling to $100 \mathrm{~K}$ and below can drastically reduce the detrimental effects of radiation damage (Garman \& Owen, 2006; Meents et al., 2010; Rodgers, 1994). Macromolecular crystals typically contain $30-90 \%$ solvent (predominantly water) in channels and are surrounded by their mother liquor. Direct cooling of these crystals leads to the formation of hexagonal ice, which disrupts the crystal lattice. Successful cryocooling of macromolecular crystals therefore requires the conversion of the water to amorphous ice, also referred to as vitrification.

In MX, vitrification is normally achieved by exposing the crystals to penetrating cryoprotectants such as glycerol or ethylene glycol prior to flash-cooling (Garman \& Schneider, 1997; Hope, 1988). However, finding optimal cryoconditions is often challenging and timeconsuming and can require a large number of crystals. Even though successful cryoprotection of systems with weak crystal contacts such as viruses has been reported in several cases (see, for example, Gan $e t$ al., 2006; Nam et al., 2007; Xiao et al., 2005), there are many systems for which it has proved impossible to find suitable cryoconditions and many experiments are still performed at room temperature. Inspection of the VIPER database (http://viperdb.scripps.edu) indicates that the large majority of virus structures have been determined at room temperature (Carrillo-Tripp et al., 2009).

The same problem occurs in the cryo-electron microscopy (cryoEM) of biological samples. Here, cooling to cryogenic temperatures is required to work under vacuum conditions and also to reduce radiation damage as in the case of macromolecular crystals. Similar to those in MX, cryo-EM samples also possess a high water content, but cryoprotectants are preferably avoided as they can alter the structure of the samples. A commonly applied technique in biological cryo-EM is high-pressure-freezing (HPF). HPF does not require any cryoprotectants and allows vitrification of the samples in their native state (Moor, 1971). For HPF, samples are first pressurized to $210 \mathrm{MPa}$ and then immediately cooled to $77 \mathrm{~K}$ with liquid nitrogen (Hohenberg et al., 1994; Studer et al., 1995, 2008). Using this technique, cooling rates of up to $7000 \mathrm{~K} \mathrm{~s}^{-1}$ can be achieved (Shimoni \& Müller, 1998).

HPF was introduced to MX in 1973 (Thomanek et al., 1973) and was further developed by Kim et al. (2005). In contrast to the method routinely applied in cryo-EM, the protocol involves slow pressurization and cooling rates below $2 \mathrm{~K} \mathrm{~s}^{-1}$ (Urayama et al., 2002). Structures of several protein crystals have been determined using this approach (Albright et al., 2006; Barstow et al., 2008; Chen et al., 2009; Collins et al., 2005, 2011; Domsic et al., 2008; Kim et al., 2006). 


\section{short communications}

However, the successful HPF of challenging systems such as viruses has not been reported until now.

Recently, an alternative approach for HPF of macromolecular crystals was developed by our group and subsequently by Kurz and coworkers (Burkhardt et al., 2012; Kurz et al., 2012). Our method involves surface-cooling rates of $3 \times 10^{3}$ to $2 \times 10^{4} \mathrm{~K} \mathrm{~s}^{-1}$, which are typically required for vitrification of biological samples using a commercially available device (Bald, 1986; Riehle, 1968). It was the goal of the present work to determine whether this protocol can be applied to sensitive crystals of a large unit-cell system with weak crystal contacts, for which often no cryoconditions can be established.

\section{Experimental}

\subsection{Crystal growth}

Crystals of bovine enterovirus 2 (BEV2; grown and purified in the Department of Microbiology, University of Leeds by Professor D. Rowlands) were grown from virus at a concentration of $7 \mathrm{mg} \mathrm{ml}^{-1}$ in 96-well Greiner CrystalQuick SW and CrystalQuick X plates using a Cartesian robot (Walter et al., 2005). The trays were sealed with Greiner VIEWseal tape and stored at $293.5 \mathrm{~K}$. Crystals of bipyramidal morphology grew in many conditions from the SaltRx screen (Hampton Research) which did not contain any cryoprotectant (Axford et al., 2012).

\subsection{High-pressure-freezing}

Sample preparation, high-pressure-freezing and subsequent sample manipulation were performed as reported previously (Burkhardt et al., 2012). BEV2 crystals with dimensions of less than $50 \mu \mathrm{m}$ were drawn into thin-walled glass capillaries with an inner diameter of $\sim 110 \mu \mathrm{m}$ (see Fig. 1). The capillaries containing the virus crystals were cut into smaller pieces, sandwiched between two aluminium platelets and then subjected to HPF using a Baltec HPM 010 device (for a more detailed description, see Dahl \& Staehelin, 1989). After HPF, the sample was immediately transferred into a liquid-nitrogen bath. Further sample manipulation was performed in an AFS2 freeze substitution device (Leica) at cryogenic temperatures below $135 \mathrm{~K}$. Unlike in previous experiments, the capillary segments

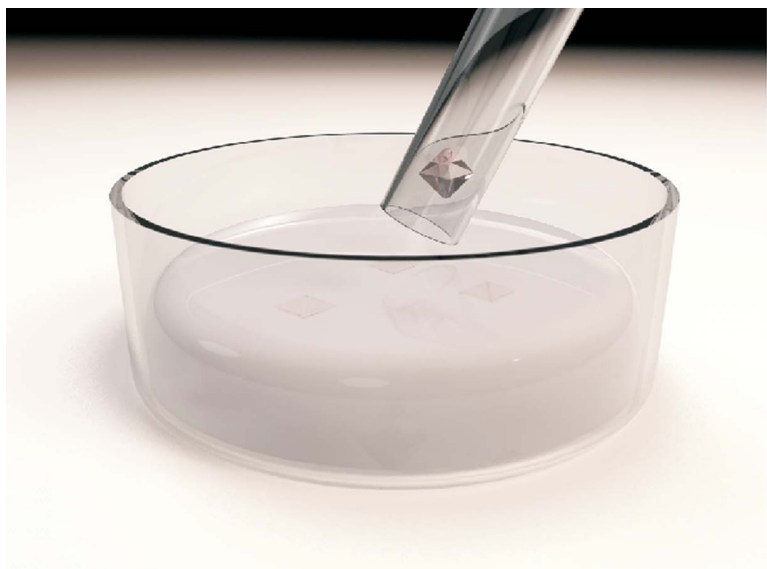

(a)

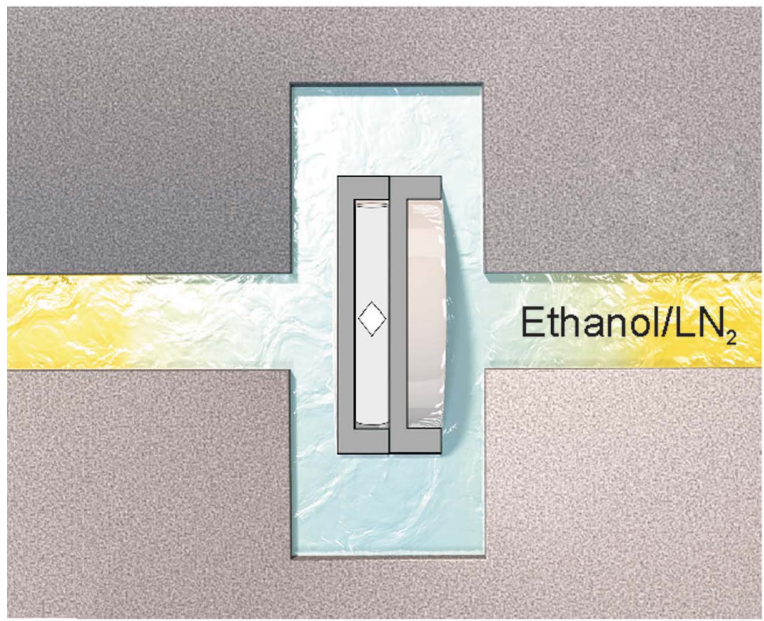

(c)
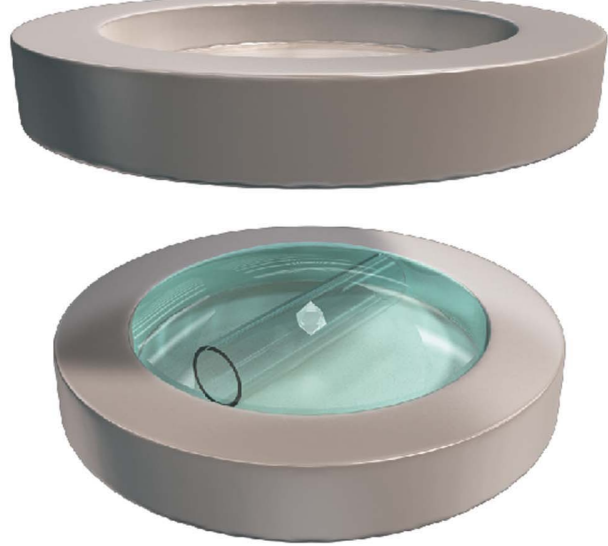

(b)

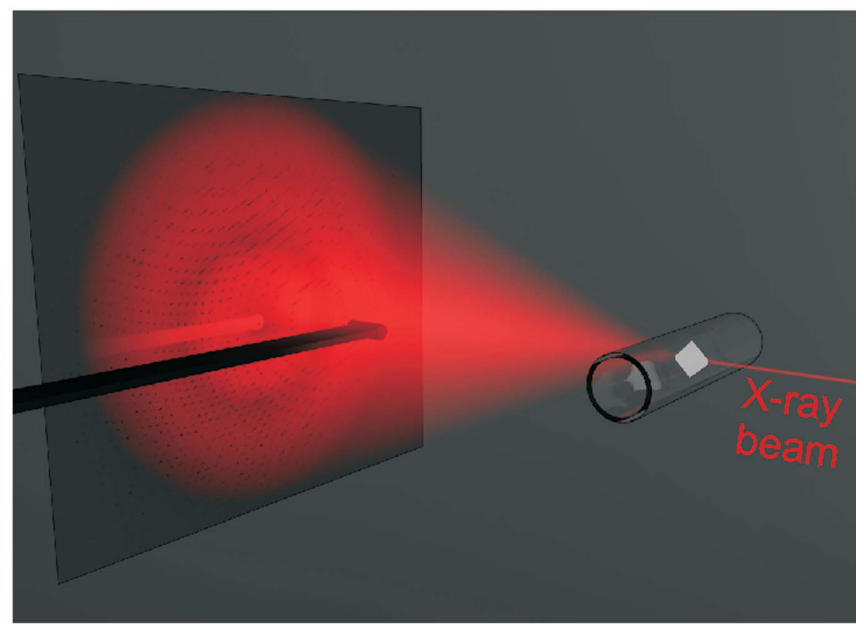

(d)

Figure 1

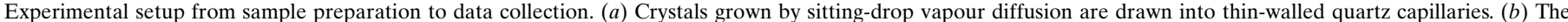

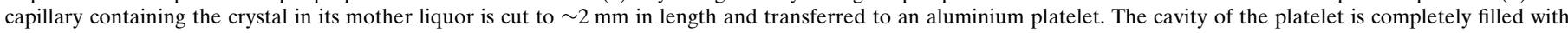

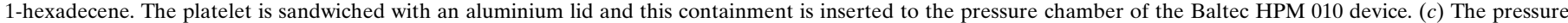

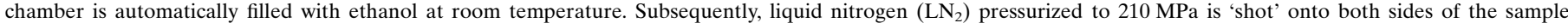

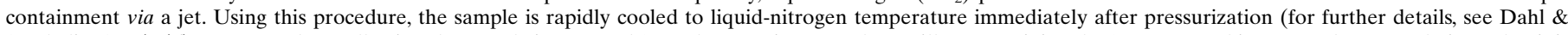

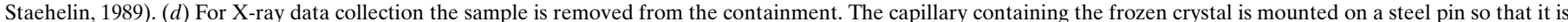
oriented perpendicular to the incident X-ray beam. All of these sample-handling procedures are performed at cryogenic temperatures. 
were inserted at one end into a hollow steel pin with an inner diameter of $210 \mu \mathrm{m}$ and fixed. The steel pin itself was mounted on a standard magnetic crystal cap (Hampton Research).

\subsection{Data collection, data processing and structure refinement}

Diffraction data from the HPF BEV2 crystal were collected on the micro-focus beamline I24 at the Diamond Light Source (DLS), Didcot, England using a Pilatus 6M detector (Dectris, Switzerland). A total of 150 images were collected using an X-ray wavelength of $0.9686 \AA$, a rotation range of $0.2^{\circ}$ per image and an exposure time of $0.2 \mathrm{~s}$ per frame. The crystal-to-detector distance was $450 \mathrm{~mm}$. The beamsize was adjusted to $10 \times 10 \mu \mathrm{m}$. Diffraction data were collected at $100 \mathrm{~K}$ using an open-flow nitrogen cryostat. Data collection from cryoprotected BEV2 crystals was performed on beamline I03 at DLS (Table 1).

Diffraction data at room temperature $(293.5 \mathrm{~K})$ were also collected on beamline I24 using a Pilatus $6 \mathrm{M}$ detector. A total of 326 images were collected from 28 crystals at 76 crystal positions using an X-ray wavelength of $0.9778 \AA$, a rotation range of $0.1^{\circ}$ per image and an exposure time of $0.1 \mathrm{~s}$ per frame. The crystal-to-detector distance was 482 or $530 \mathrm{~mm}$. The beamsize was adjusted to $20 \times 20 \mu \mathrm{m}$.

Data frames were indexed and integrated with $H K L-2000$ and scaled with SCALEPACK (Otwinowski \& Minor, 1997). Structure refinements were performed with CNS using strict fivefold NCS constraints (Brünger et al., 1998).

\section{Results and discussion}

We describe the first successful structure determination from highpressure-frozen virus crystals. We have developed a protocol which allows high-pressure-freezing at high cooling rates using a commercially available HPF device (Baltec HPM 010). Crystals of bovine enterovirus 2 (BEV2), a virus requiring no disease precautions, were grown by sitting-drop vapour diffusion (Walter et al., 2005). The $\mathrm{BEV} 2$ crystals belonged to space group $F 23$, with unit-cell parameter

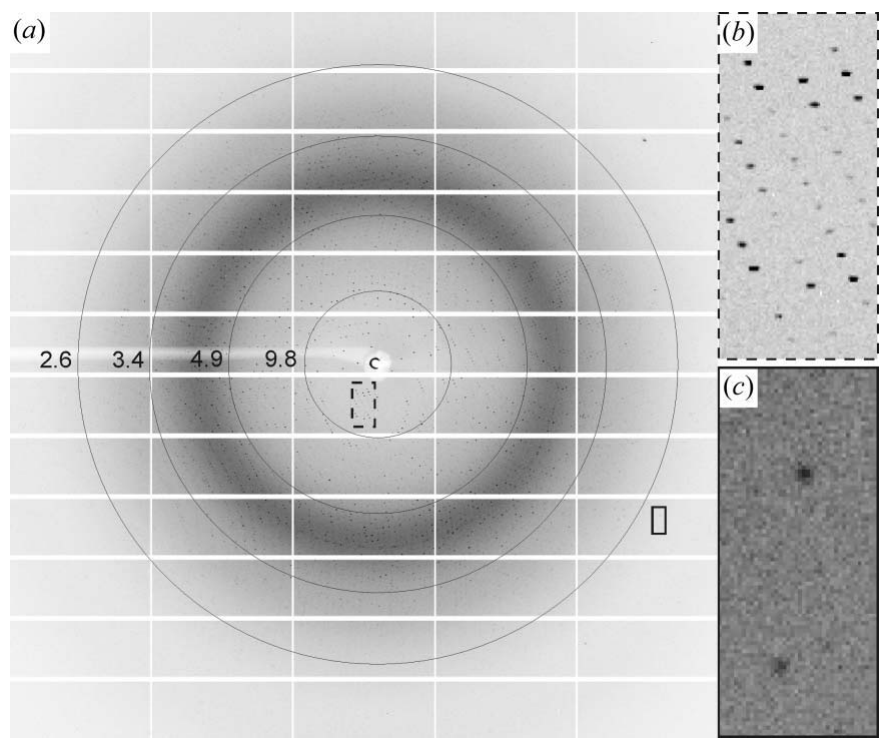

\section{Figure 2}

Diffraction data collected from an HPF BEV2 crystal on the micro-focus beamline I24 at the Diamond Light Source (rotation increment $0.2^{\circ}$ ). (a) Overall diffraction image. (b) Enlargement showing low-resolution data between 11.4 and $27.1 \AA$ resolution [rectangle with dashed line in $(a)$ ]. (c) Enlargement showing highresolution data around $2.4 \AA$ resolution [rectangle with solid line in $(a)$ ].
Table 1

Data-collection and refinement statistics.

Values in parentheses are for the highest resolution shell.

\begin{tabular}{|c|c|c|c|}
\hline & $\mathrm{HPF}$ & RT & $\mathrm{CP} \dagger$ \\
\hline \multicolumn{4}{|l|}{ Data collection } \\
\hline Crystal size $(\mu \mathrm{m})$ & $30 \times 30 \times 30$ & $\begin{array}{l}50 \times 50 \times 50 \text { to } \\
100 \times 100 \times 100\end{array}$ & $50 \times 50 \times 50$ \\
\hline Temperature (K) & 100 & 293.5 & 100 \\
\hline Unit-cell parameter $(\AA)$ & $a=432.5$ & $a=436.6$ & $a=426.5$ \\
\hline Space group & $F 23$ & $F 23$ & $F 23$ \\
\hline Resolution $(\AA)$ & $\begin{array}{l}50.0-2.50 \\
\quad(2.59-2.50)\end{array}$ & $\begin{array}{l}50.0-2.10 \\
\quad(2.18-2.10)\end{array}$ & $\begin{array}{l}50.0-4.00 \\
\quad(4.14-4.00)\end{array}$ \\
\hline No. of observed reflections & 281645 & 592107 & 163227 \\
\hline No. of unique reflections & 163062 & 260688 & 47613 \\
\hline$R_{\text {merge }}(\%)$ & $27.5(-)$ & $19.6(40.4)$ & $36.7(-)$ \\
\hline Completeness (\%) & $71.6(74.4)$ & $65.8(17.2)$ & $88.6(91.5)$ \\
\hline$\langle I / \sigma(I)\rangle$ & $2.2(0.5)$ & $4.3(1.2)$ & $2.6(0.7)$ \\
\hline Multiplicity & $1.7(1.6)$ & $2.3(1.2)$ & $3.4(3.3)$ \\
\hline Mosaic spread $\left({ }^{\circ}\right)$ & 0.26 & $\sim 0.04$ & $\sim 0.70$ \\
\hline \multicolumn{4}{|l|}{ Refinement } \\
\hline Resolution range $(\AA)$ & $50-2.50$ & $50-2.10$ & \\
\hline No. of reflections & 157703 & 247641 & \\
\hline No. of protein residues & 6224 & 6224 & \\
\hline No. of ligand molecules & 42 & 42 & \\
\hline No. of water molecules & 267 & 289 & \\
\hline R.m.s.d. bond lengths $(\AA)$ & 0.010 & 0.012 & \\
\hline R.m.s.d. bond angles $\left({ }^{\circ}\right)$ & 1.6 & 1.7 & \\
\hline$R_{\text {work }}(\%)$ & 22.7 & 19.6 & \\
\hline$R_{\text {free }}+(\%)$ & 23.5 & 20.7 & \\
\hline \multicolumn{4}{|l|}{ Average $B$ factors $\left(\AA^{2}\right)$} \\
\hline Protein atoms & 26 & 17 & \\
\hline Ligand atoms & 47 & 35 & \\
\hline Water molecules & 35 & 26 & \\
\hline Average particle radius $(\AA)$ & 127.7 & 128.9 & \\
\hline
\end{tabular}

$\dagger$ Cryoprotected. $\ddagger R_{\text {free }}$ was calculated from $1 \%$ of the data that were randomly chosen and omitted from refinement; however, it is of limited significance owing to the considerable noncrystallographic symmetry.

$a=436.6 \AA$ at room temperature. Initial attempts to cryoprotect these BEV2 crystals using several standard procedures with glycerol, ethylene glycol and paraffin oil failed to yield useful diffraction data owing to an increase in the mosaic spread and a dramatic decrease in the resolution of diffraction (from $2.1 \AA$ at room temperature to no better than $4.0 \AA$ at cryogenic temperatures; Table 1).

Fig. 1 shows our experimental setup for data collection from frozen crystals, including sample preparation, HPF and X-ray diffraction measurements.

HPF BEV2 crystals diffracted to reasonable resolution with clearly separated diffraction spots (Fig. 2) and possessed mosaic spreads of about $0.26^{\circ}$. This is much smaller than that observed for conventionally cryoprotected crystals and sufficiently small as to not impede data collection from crystals with large unit cells as in our case. Using a focused beamsize of $10 \times 10 \mu \mathrm{m}$, the crystals could withstand the unattenuated X-ray beam for about $12 \mathrm{~s}$ before showing severe signs of radiation damage. A data set obtained from one single crystal of $30 \mu \mathrm{m}$ in all dimensions was used for subsequent crystal structure refinement.

For direct comparison, room-temperature (RT) diffraction data were collected from BEV2 crystals on the same beamline. At room temperature the crystal lifetime with a less focused beam of $20 \times$ $20 \mu \mathrm{m}$ varied between 0.4 and $0.6 \mathrm{~s}$. A total of 326 diffraction images were collected at 76 different crystal positions from 28 individual crystals with sizes between 50 and $100 \mu \mathrm{m}$ by in situ diffraction from a single crystallization plate (Axford et al., 2012). Note that these crystals were significantly larger (on average about twice as large in each direction) than that subjected to HPF. All room-temperature diffraction images were merged into one data set for structure refinement. Data-collection parameters and refinement statistics of both the HPF crystal data set and the merged RT data set are 


\section{short communications}
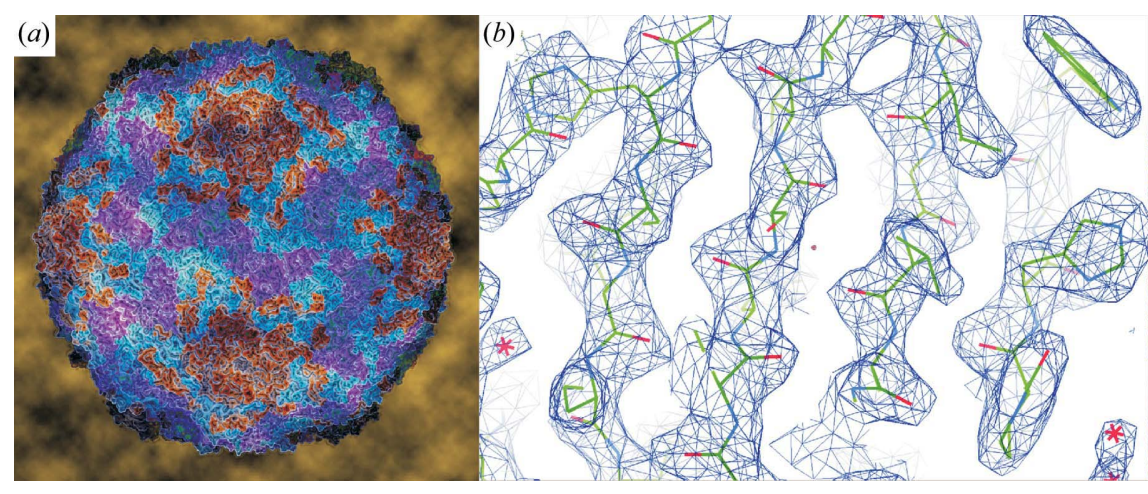

(c)

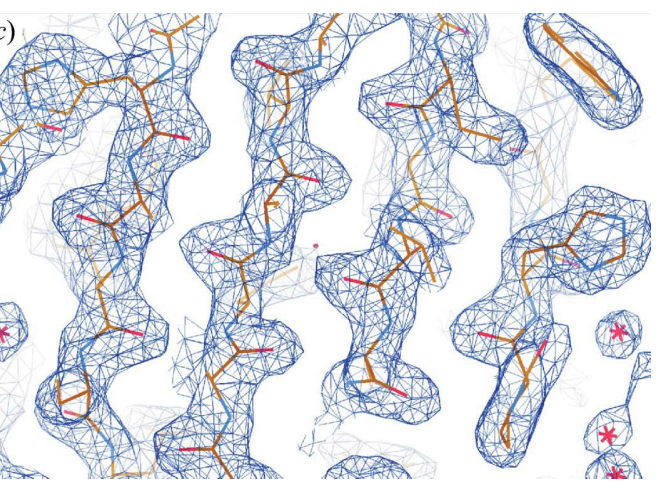

Figure 3

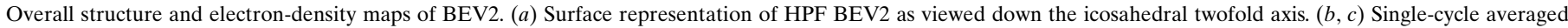

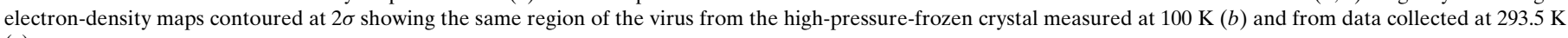
(c).

summarized in Table 1. For direct comparison of HPF with conventional cryoprotection (CP), Table 1 also shows low-temperature data from a crystal cryoprotected with $25 \%$ glycerol. Owing to the poor diffraction properties of the crystal, structure refinement was not possible.

Fig. 3(a) shows the electron density obtained from a single highpressure-frozen BEV2 crystal. The HPF and RT structures are very similar at the detailed level (r.m.s. deviation of $0.29 \AA$ in $\mathrm{C}^{\alpha}$ atoms when the complete icosahedral asymmetric units are superposed as a single rigid body), although there is an $\sim 1 \%$ shrinkage in the unit cell and in the overall size of the virus particle on cooling (Table 1). The electron-density maps possess similar features and are of comparable quality (Figs. $3 b$ and $3 c$ ).

Overall, the quality of the data is similar despite the HPF data being recorded at a single position from one crystal rather than at 76 positions from 28 larger crystals as in the case of the roomtemperature measurements. Taking the fourfold higher flux density and the smaller dimensions of the HPF crystal into account, the 'gain factor' in radiation tolerance for data collection at $100 \mathrm{~K}$ compared with room-temperature measurements is more than 100 in our case.

In this work, we demonstrate successful HPF of virus crystals for the first time. HPF is very well suited to the successful cryocooling of large unit-cell systems such as BEV2. All of the equipment used in this study is commercially available and there is still great scope for optimizing the logistics of sample handling and data collection to improve the quality of the HPF data and the success rate of the method. Not only is data collection feasible from far fewer crystals, but for large viruses, for which only a single image can be recorded per crystal at room temperature, this approach will increase the effective resolution obtainable and is likely to render some otherwise almost impossible projects tractable (Abrescia et al., 2004, 2008).

Our virus crystals were directly frozen from their mother liquor without the need for any penetrative cryoprotectants. This makes HPF a method that is useful not only for the special case of virus crystallography but also for challenging problems in macromolecular crystallography in general. HPF will allow cryogenic data collection from all macromolecular crystals independent of their growth conditions. This method should have a large impact as it allows data collection from frozen crystals without the time-consuming and also crystal-consuming search for optimal cryoconditions.

We would like to thank Professor D. Rowlands (University of Leeds) for providing samples of BEV2. DIS and EEF are supported by the MRC and JR by the Wellcome Trust. M. Holthaus is acknowledged for providing illustrations of the HPF setup.

\section{References}

Abrescia, N. G., Cockburn, J. J., Grimes, J. M., Sutton, G. C., Diprose, J. M., Butcher, S. J., Fuller, S. D., San Martín, C., Burnett, R. M., Stuart, D. I., Bamford, D. H. \& Bamford, J. K. (2004). Nature (London), 432, 68-74

Abrescia, N. G., Grimes, J. M., Kivelä, H. M., Assenberg, R., Sutton, G. C., Butcher, S. J., Bamford, J. K., Bamford, D. H. \& Stuart, D. I. (2008). Mol. Cell, 31, 749-761.

Albright, R. A., Ibar, J. L., Kim, C. U., Gruner, S. M. \& Morais-Cabral, J. H. (2006). Cell, 126, 1147-1159.

Axford, D. et al. (2012). Acta Cryst. D68, 592-600.

Bald, W. B. (1986). J. Microsc. 143, 89-102.

Barstow, B., Ando, N., Kim, C. U. \& Gruner, S. M. (2008). Proc. Natl Acad. Sci. USA, 105, 13362-13366.

Blake, C. F. F. \& Phillips, D. C. (1962). Biological Effects of Ionizing Radiation at the Molecular Level, pp. 183-191. Vienna: IAEA.

Brünger, A. T., Adams, P. D., Clore, G. M., DeLano, W. L., Gros, P., GrosseKunstleve, R. W., Jiang, J.-S., Kuszewski, J., Nilges, M., Pannu, N. S., Read, R. J., Rice, L. M., Simonson, T. \& Warren, G. L. (1998). Acta Cryst. D54, 905-921.

Burkhardt, A., Warmer, M., Panneerselvam, S., Wagner, A., Zouni, A., Glöckner, C., Reimer, R., Hohenberg, H. \& Meents, A. (2012). Acta Cryst. F68, 495-500.

Carrillo-Tripp, M., Shepherd, C. M., Borelli, I. A., Venkataraman, S., Lander, G., Natarajan, P., Johnson, J. E., Brooks, C. L. \& Reddy, V. S. (2009). Nucleic Acids Res. 37, D436-D442.

Chen, Y.-F., Tate, M. W. \& Gruner, S. M. (2009). J. Appl. Cryst. 42, 525-530.

Collins, M. D., Hummer, G., Quillin, M. L., Matthews, B. W. \& Gruner, S. M. (2005). Proc. Natl Acad. Sci. USA, 102, 16668-16671.

Collins, M. D., Kim, C. U. \& Gruner, S. M. (2011). Annu. Rev. Biophys. 40, 81-98.

Dahl, R. \& Staehelin, L. A. (1989). J. Electron Microsc. Tech. 13,165-174.

Domsic, J. F., Avvaru, B. S., Kim, C. U., Gruner, S. M., Agbandje-McKenna, M., Silverman, D. N. \& McKenna, R. (2008). J. Biol. Chem. 283, 30766-30771.

Gan, L., Speir, J. A., Conway, J. F., Lander, G., Cheng, N., Firek, B. A., Hendrix, R. W., Duda, R. L., Liljas, L. \& Johnson, J. E. (2006). Structure, 14, 16551665.

Garman, E. F. \& Owen, R. L. (2006). Acta Cryst. D62, 32-47.

Garman, E. F. \& Schneider, T. R. (1997). J. Appl. Cryst. 30, 211-237.

Hohenberg, H., Mannweiler, K. \& Müller, M. (1994). J. Microsc. 175, 34-43.

Hope, H. (1988). Acta Cryst. B44, 22-26.

Kim, C. U., Hao, Q. \& Gruner, S. M. (2006). Acta Cryst. D62, 687-694.

Kim, C. U., Kapfer, R. \& Gruner, S. M. (2005). Acta Cryst. D61, 881-890.

Kurz, M., Blattmann, B., Kaech, A., Briand, C., Reardon, P., Ziegler, U. \& Gruetter, M. G. (2012). J. Appl. Cryst. 45, 999-1008.

Meents, A., Gutmann, S., Wagner, A. \& Schulze-Briese, C. (2010). Proc. Natl Acad. Sci. USA, 107, 1094-1099.

Moor, H. (1971). Philos. Trans. R. Soc. Lond. B, 261, 121-131. 


\section{short communications}

Nam, H.-J., Lane, M. D., Padron, E., Gurda, B., McKenna, R., Kohlbrenner, E., Aslanidi, G., Byrne, B., Muzyczka, N., Zolotukhin, S. \& AgbandjeMcKenna, M. (2007). J. Virol. 81, 12260-12271.

Otwinowski, Z. \& Minor, W. (1997). Methods Enzymol. 276, 307-326.

Riehle, U. (1968). Chem. Ing. Tech. 40, 213-218.

Rodgers, D. W. (1994). Structure, 2, 1135-1140.

Shimoni, E. \& Müller, M. (1998). J. Microsc. 192, 236-247.

Studer, D., Humbel, B. M. \& Chiquet, M. (2008). Histochem. Cell Biol. 130, $877-889$.
Studer, D., Michel, M., Wohlwend, M., Hunziker, E. B. \& Buschmann, M. D. (1995). J. Microsc. 179, 321-332.

Thomanek, U. F., Parak, F., Mössbauer, R. L., Formanek, H., Schwager, P. \& Hoppe, W. (1973). Acta Cryst. A29, 263-265.

Urayama, P., Phillips, G. N. \& Gruner, S. M. (2002). Structure, 10, 51-60.

Walter, T. S. et al. (2005). Acta Cryst. D61, 651-657.

Xiao, C., Bator-Kelly, C. M., Rieder, E., Chipman, P. R., Craig, A., Kuhn, R. J., Wimmer, E. \& Rossmann, M. G. (2005). Structure, 13, 10191033. 